\title{
A Non-linear Estimation of the Capital Asset Pricing Model: The Case of Japanese Automobile Industry Firms
}

\author{
Chikashi Tsuji ${ }^{1}$ \\ ${ }^{1}$ Faculty of Economics, Chuo University, Tokyo, Japan \\ Correspondence: Chikashi Tsuji, Professor, Faculty of Economics, Chuo University, 742-1 Higashinakano, Hachioji-shi, \\ Tokyo 192-0393, Japan.
}

Received: March 14, 2017

Accepted: March 30, 2017

Available online: April 5, 2017

doi:10.11114/afa.v3i2.2331

URL: https://doi.org/10.11114/afa.v3i2.2331

\begin{abstract}
This paper quantitatively examines a non-linear capital asset pricing model (CAPM) by using monthly stock returns of major automobile industry firms in Japan. Applying the maximum likelihood method, we derive the following interesting findings. (1) First, in the case where the distribution of stock returns has a fat-tail, our non-linear CAPM is highly effective. Because the parameters of our non-linear CAPM well capture fat-tailed return distributions, the non-linear model estimation derives reliable estimates of beta values. (2) Second, in the case where stock returns are normally distributed, our non-linear CAPM is also effective. Since the parameters of our non-linear CAPM also well capture normally distributed returns by adjusting its degrees of freedom parameter value, the non-linear model estimation similarly derives reliable beta estimates as those derived from the standard linear CAPM. (3) Finally, we further conduct the Wald tests based on the estimators from the standard CAPM and our non-linear CAPM, and we suggest that in the case where the distribution of stock returns has a fat-tail, the Wald test based on the estimators from our non-linear CAPM shall be more reliable than the Wald test based on the estimators from the standard linear CAPM.
\end{abstract}

Keywords: asset pricing, beta, CAPM, fat-tail, Japanese stock market, maximum likelihood method

\section{Introduction}

The capital asset pricing model (CAPM) is one of the most famous asset pricing models in finance (see, e.g., Sharpe, 1964). Hence, many studies of stock returns by applying this model have been conducted by academicians and this model is also often used in practice as a basic benchmark model. The application of this model is generally conducted by such a linear regression approach as the ordinary least squares (OLS) method; and this method generally assumes a normal distribution for stock returns. However, in fact, stock returns often follow fatter-tailed distributions, thus more careful methods for the CAPM estimation incorporating fat-tailed stock return distributions are needed. Based on this motivation, this paper quantitatively examines a non-linear CAPM by using monthly stock returns of major automobile industry firms in Japan; and this is the objective of this study.

Applying the maximum likelihood method, this study derives the following interesting findings. (1) First, in the case where the distribution of stock returns has a fat-tail, our non-linear CAPM is highly effective. Since the parameters of our non-linear CAPM well capture heavy-tailed stock return distributions, the non-linear model derives reliable estimates of the CAPM beta values. (2) Second, in the case where stock returns are normally distributed, our non-linear CAPM is also effective. Because the parameters of our non-linear CAPM also capture normally distributed returns by adjusting its degrees of freedom (DOF) parameter value, the non-linear model similarly obtains reliable beta estimates as the standard linear CAPM. (3) Moreover, we further conduct the Wald tests based on the estimators from the standard CAPM and a non-linear CAPM; and we suggest that in the case where the distribution of stock returns has a fat-tail, the Wald test result based on the estimators from our non-linear CAPM shall be more reliable than the Wald test result based on the estimators from the standard CAPM. These interesting and useful findings are the contributions of this study. Regarding the rest of this paper, in Section 2, we review recent related studies; in Section 3, we explain our data and variables; and in Section 4, we document our quantitative methodology. After these, Section 5 explains our results and Section 6 summarizes the paper.

\section{Literature Review}

This section briefly reviews recent literature. Recently, Messis and Zapranis (2014) studied the existence of herding 
phenomena, coskewness, and cokurtosis for French, German, UK, US, and Chinese stock markets by using the CAPM framework. Employing the framework of a fuzzy set theory and fuzzy returns, Moussa et al. (2014) analyzed the CAPM betas via the fuzzy least square method.

Arısoy et al. (2015) suggested a volatility-based CAPM, where asset betas change in response to the changes in investors' expectations as to near-term aggregate volatility. De Moor and Sercu (2015) suggested that the extreme observations can lead the bias for the average stock return calculations; and in order to examine the impact of extreme observations on alphas of the CAPM, they tested several filters that could help to decrease such a bias.

Vendrame et al. (2016) also considered some extensions of the CAPM. More concretely, they examined the conditional models, which used individual stocks rather than portfolios. They also examined the models that were extended to include the higher moments and factor dimensions. Further, a study by Tsuji (2012) empirically tested the portfolio alphas in Japan with comparing the similar portfolios in the US.

\section{Data and Variables}

This section documents the data used in this study. Our data are monthly and all data are from Thomson Reuters. First, subtracting the Japanese three-month interbank rate from the return of the Tokyo stock price index (TOPIX), we derive monthly excess percentage returns of the Japanese stock market. Next, using the same interest rate and stock price data of Japanese automobile industry firms, we compute their monthly excess returns. More concretely, we derive and use monthly excess percentage returns of six Japanese automobile industry firms of Toyota Motor Corporation, Nissan Motor Co., Ltd., Suzuki Motor Corporation, Mitsubishi Motors Corporation, Mazda Motor Corporation, and Honda Motor Co., Ltd. Thus, in this study, we use the above seven excess percentage stock return series; and the sample period of our data is from January 1989 to February 2017. We consider that employing these Japanese major automobile industry firms is useful and meaningful for the international stock market case study.

Table 1 shows the descriptive statistics for the excess percentage returns of the Japanese stock market and the excess percentage returns of the Japanese six automobile industry firms. We note that the Jarque-Bera tests statistically significantly reject the normality of all the excess returns except for the case of Honda. This interestingly means that during our sample period, only the excess stock return of Honda follows a normal distribution. In addition, the kurtosis values in Table 1 also suggest that the other six excess returns follow fat-tailed distributions. Further, the Jarque-Bera statistic is particularly large in the case of Mitsubishi (423.9641 as in Table 1) and this means that the stock return of Mitsubishi has the fattest-tailed distribution in our samples. We therefore emphasize that it is interesting to note and compare the estimation results of linear and non-linear CAPMs by focusing on the cases of Honda and Mitsubishi. Moreover, Figure 1 displays the scatter plots of the six pairs of monthly excess stock market returns and monthly excess stock returns of the six automobile industry firms in Japan.

Table 1. Descriptive statistics for excess returns of the Japanese stock market and six automobile industry firms in Japan: For the period from January 1989 to February 2017

\begin{tabular}{lllll}
\hline & Market & Toyota & Nissan & Suzuki \\
\hline Mean & -0.0807 & 0.4947 & 0.3311 & 0.7722 \\
Median & -0.0056 & 0.1975 & -0.0468 & 0.8770 \\
SD & 5.6128 & 7.3765 & 9.6838 & 8.3294 \\
Skewness & -0.1712 & 0.6905 & 0.2120 & -0.1838 \\
Kurtosis & 3.8142 & 6.1444 & 4.8963 & 3.6911 \\
JB stat. & $10.9876^{* * *}$ & $166.1031^{* * *}$ & $53.1757^{* * *}$ & $8.6296^{* *}$ \\
$p$-value & 0.0041 & 0.0000 & 0.0000 & 0.0134 \\
\hline Mean & Mitsubishi & Mazda & Honda \\
Median & -0.3239 & 0.3615 & 0.5904 \\
SD & -1.1650 & -0.0158 & & 0.3754 \\
Skewness & 11.0562 & 12.0609 & & 8.2128 \\
Kurtosis & 0.5714 & 0.2811 & & 0.0186 \\
JB stat. & 8.3664 & 4.6472 & & 3.4841 \\
$p$-value & $423.9641^{* * *}$ & $42.6611^{* * *}$ & & 3.3203 \\
\hline
\end{tabular}

Notes: $* * *$ and $* *$ denote the statistical significance at the $1 \%$ and $5 \%$ levels, respectively. In addition, JB stat. means the Jarque-Bera statistic, SD denotes the standard deviation value, and the number of our observations is 338 . 


\section{Methodology}

We first estimate the following standard CAPM (1) by the ordinary least squares (OLS) method.

$$
r_{i t}-r_{f t}=\alpha_{i}+\beta_{i}\left(r_{m t}-r_{f t}\right)+u_{i t}, \quad u_{i t} \sim i i d N\left(0, \sigma_{i}^{2}\right) .
$$

As seen in the equation (1), in this paper, $r_{i t}-r_{f t}$ means the excess percentage return of one of the Japanese six automobile industry firms documented in the previous section. Further, $r_{m t}-r_{f t}$ denotes the excess percentage return of the Japanese stock market and $\beta_{i}$ means the so-called beta value of the CAPM. In the model (1), the residuals are assumed to follow an independent and identically distributed (iid) normal distribution, whose standard deviation is $\sigma_{i}$.

Next, by the maximum likelihood (ML) method, we estimate the following non-linear CAPM (2).

$$
r_{i t}-r_{f t}=\alpha_{i}+\beta_{i}\left(r_{m t}-r_{f t}\right)+\sigma_{i} \sqrt{\frac{v_{i}-2}{v_{i}}} \kappa_{i t} .
$$

In the above equation (2), the disturbance term $\kappa_{i t}$ follows a Student's $t$ distribution, and $v_{i}$ represents the degrees of freedom (DOF) parameter (These two are the same in the below equation (3) and $\sigma_{i}$ always denotes the standard deviation.). In order to estimate this non-linear CAPM (2), we maximize the following log likelihood function:

$$
\ln l_{t}\left(\theta_{i}\right)=\ln \left(\frac{\Gamma\left(\frac{v_{i}+1}{2}\right)}{\sqrt{\pi v_{i}} \Gamma\left(\frac{v_{i}}{2}\right)}\right)-\frac{v_{i}+1}{2} \ln \left(1+\frac{\kappa_{i t}^{2}}{v_{i}}\right)-\ln \sigma_{i}+\ln \sqrt{\frac{v_{i}}{v_{i}-2}} .
$$

In this log likelihood function $(3), \Gamma(\cdot)$ indicates the gamma function.

Finally, we also perform the Wald test. Its null hypothesis is $\beta_{i}=1$; and the alternative hypothesis is $\beta_{i} \neq 1$. If $\beta_{i}=1$, it is considered that the company's stock return behaves in a similar manner as the overall Japanese stock market return.

\section{Results}

As we previously noted, it is interesting to pay attention to the differences of the linear and non-linear CAPM estimation results by focusing on the cases of Mitsubishi and Honda. We also inspect the Wald test results for the two firms. First, Table 2 shows that the standard CAPMs (1) are successfully estimated by the OLS method. As in Table 2, all beta values for the models are statistically significantly estimated. The estimated beta values are 0.8324 for Toyota, 0.9941 for Nissan, 0.8895 for Suzuki, 0.9087 for Mitsubishi, 1.3702 for Mazda, and 0.8264 for Honda, respectively.

Next, Table 3 suggests that our non-linear CAPMs (2) are successfully estimated by the ML method. As exhibited in Table 3, all beta values and DOF parameters in our non-linear CAPMs are statistically significantly estimated. The estimated beta values are 0.8174 for Toyota, 0.9900 for Nissan, 0.9218 for Suzuki, 0.7816 for Mitsubishi, 1.3027 for Mazda, and 0.8463 for Honda, respectively. An interesting result here is that the estimated beta values of Mitsubishi are very different for two models: for the standard CAPM, it is 0.9087 , while it is 0.7816 for the non-linear CAPM. We consider that this is because of the effect of much fatter-tailed stock return distribution of Mitsubishi. In fact, as shown in Table 3, the estimated DOF parameter for Mitsubishi shows the smallest value of 2.3955, and this small DOF value means the fatter-tailed Student's $t$ distribution. In contrast, also as in Table 3, the estimated DOF parameter for Honda shows the largest value of 9.6246, indicating less fatter-tailed and roughly normal distribution. It is noted again that very interestingly, these estimation results are consistent with the results of the Jarque-Bera tests shown in Table 1.

Finally, we explain the Wald test results by focusing on the cases of Mitsubishi and Honda. First, Panel A of Table 4 shows that the Wald test result based on the OLS estimators suggests that Mitsubishi stock behaves in a similar manner as the overall stock market in Japan, while our ML estimator based Wald test result suggests the opposite. We here note that for Mitsubishi, the Wald test result based on the ML estimators should be reliable because Mitsubishi's stock return has a fatter-tailed distribution. Thus, it should be judged that Mitsubishi's stock does not behave as the overall stock market return in Japan.

On the other hand, as for Honda, the result based on the OLS estimators and that based on the ML estimators shall be both reliable because during our sample period, the stock return of Honda is normally distributed. In fact, as in Table 4, the Wald test results based on the OLS and ML estimators are the same. Namely, both results show that Honda's stock return does not behave as the overall Japanese stock market return in a statistical sense. 
Panel A. Toyota

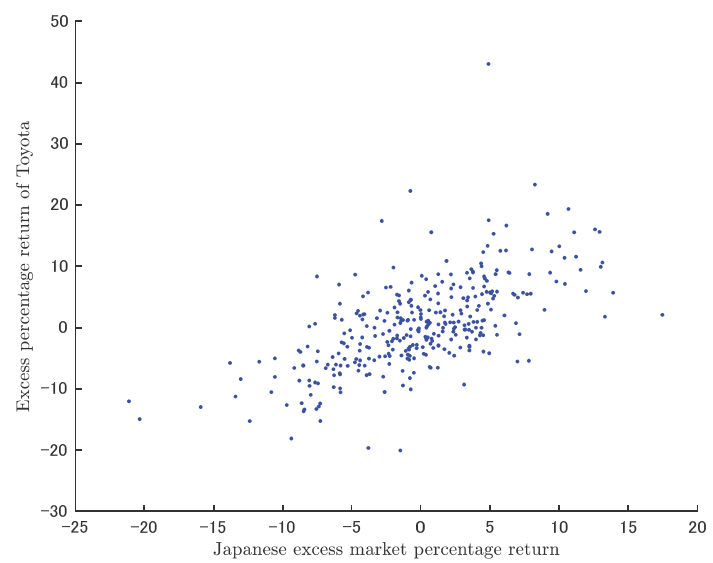

Panel C. Suzuki

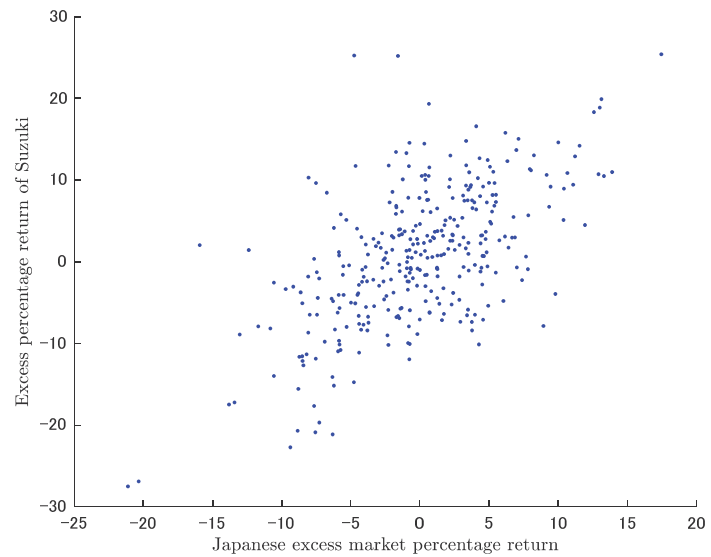

Panel E. Mazda

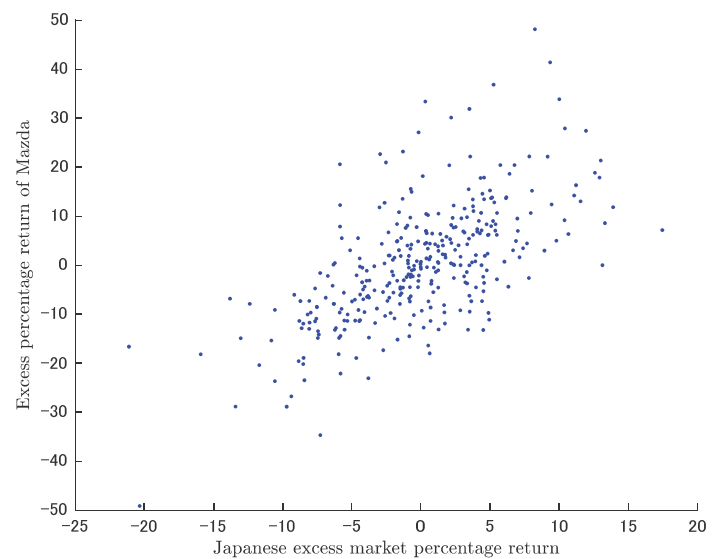

Panel B. Nissan

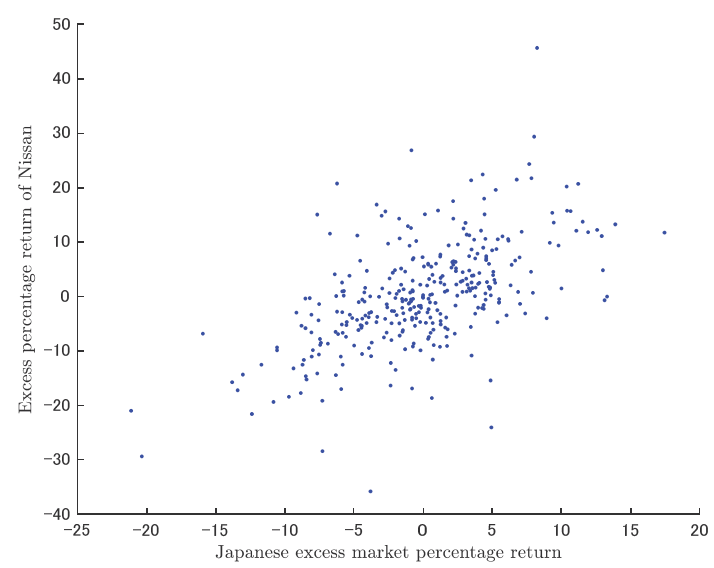

Panel D. Mitsubishi

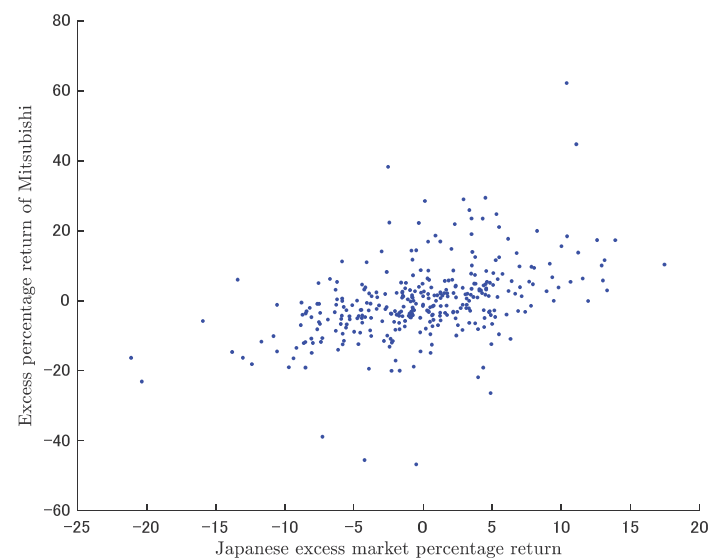

Panel F. Honda

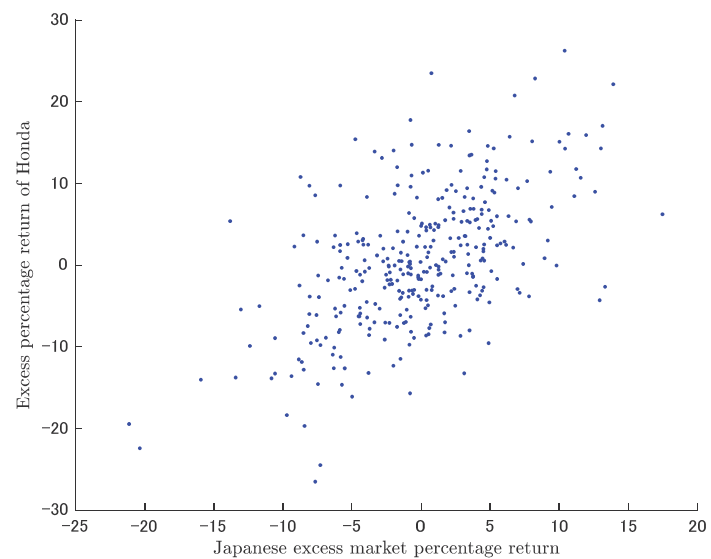

Figure 1. Scatter plots of monthly excess stock market returns and monthly excess stock returns of automobile industry firms in Japan: For the period from January 1989 to February 2017 
Table 2. OLS estimators of the standard CAPM for the Japanese major automobile industry firms: For the period from January 1989 to February 2017

\begin{tabular}{llll}
\hline & Toyota & Nissan & Suzuki \\
\hline Alpha & $0.5619^{* *}$ & 0.4114 & $0.8440^{* *}$ \\
$t$-value & 1.8120 & 0.9569 & 2.3306 \\
$p$-value & 0.0354 & 0.1696 & 0.0102 \\
Beta & $0.8324 * * *$ & $0.9941^{* * *}$ & $0.8895^{* * *}$ \\
$t$-value & 15.0463 & 12.9612 & 13.7662 \\
$p$-value & 0.0000 & 0.0000 & 0.0000 \\
\hline & Mitsubishi & Mazda & Honda \\
\hline Alpha & -0.2506 & 0.4721 & $0.6571^{* *}$ \\
$t$-value & -0.4703 & 0.9355 & 1.7848 \\
$p$-value & 0.6808 & 0.1751 & 0.0376 \\
Beta & $0.9087^{* * *}$ & $1.3702 * * *$ & $0.8264 * * *$ \\
$t$-value & 9.5584 & 15.2183 & 12.5817 \\
$p$-value & 0.0000 & 0.0000 & 0.0000 \\
\hline
\end{tabular}

Notes: This table presents the OLS estimators of the standard CAPM for the Japanese major automobile industry firms. In this table, $* * *$ and $* *$ indicate the statistical significance of the parameter at the $1 \%$ and $5 \%$ levels, respectively.

Table 3. ML estimators of a non-linear CAPM for the Japanese major automobile industry firms: For the period from January 1989 to February 2017

\begin{tabular}{|c|c|c|c|}
\hline & Toyota & Nissan & Suzuki \\
\hline Alpha & 0.2758 & 0.0012 & $0.7315 * *$ \\
\hline$t$-value & 1.0009 & 0.0033 & 2.0821 \\
\hline$p$-value & 0.1588 & 0.4987 & 0.0190 \\
\hline Beta & $0.8174 * * *$ & $0.9900 * * *$ & $0.9218 * * *$ \\
\hline$t$-value & 16.4273 & 15.0275 & 14.0688 \\
\hline$p$-value & 0.0000 & 0.0000 & 0.0000 \\
\hline Variance & $31.0394 * * *$ & $69.7180 * * *$ & $44.2700 * * *$ \\
\hline$t$-value & 7.8316 & 4.7103 & 10.4860 \\
\hline$p$-value & 0.0000 & 0.0000 & 0.0000 \\
\hline DOF & $5.0906^{* * *}$ & $3.5408 * * *$ & $9.3982 * *$ \\
\hline$t$-value & 4.0556 & 4.5230 & 2.2082 \\
\hline \multirow[t]{2}{*}{$p$-value } & 0.0000 & 0.0000 & 0.0140 \\
\hline & Mitsubishi & Mazda & Honda \\
\hline Alpha & -0.8926 & -0.0367 & $0.5074^{*}$ \\
\hline$t$-value & -2.4247 & -0.0822 & 1.3916 \\
\hline$p$-value & 0.9921 & 0.5327 & 0.0825 \\
\hline Beta & $0.7816^{* * *}$ & $1.3027 * * *$ & $0.8463 * * *$ \\
\hline$t$-value & 11.5149 & 15.1881 & 12.6952 \\
\hline$p$-value & 0.0000 & 0.0000 & 0.0000 \\
\hline Variance & $173.7875^{*}$ & $92.6435 * * *$ & $46.1964 * * *$ \\
\hline$t$-value & 1.3474 & 6.0783 & 10.4433 \\
\hline$p$-value & 0.0894 & 0.0000 & 0.0000 \\
\hline DOF & $2.3955 * * *$ & $4.1887 * * *$ & $9.6246 * *$ \\
\hline$t$-value & 6.0786 & 3.9152 & 1.7964 \\
\hline$p$-value & 0.0000 & 0.0001 & 0.0367 \\
\hline
\end{tabular}

Notes: This table presents the ML estimators of a non-linear CAPM for the Japanese major automobile industry firms. In this table, DOF denotes the degrees of freedom of a Student's $t$ distribution. Further, ***, **, and * indicate the statistical significance at the $1 \%, 5 \%$, and $10 \%$ levels, respectively. 
Table 4. Results of the Wald tests for the Japanese major automobile industry firms: For the period from January 1989 to February 2017

\begin{tabular}{|c|c|c|c|}
\hline \multicolumn{4}{|c|}{ Panel A. Results based on the OLS estimators of the standard CAPM } \\
\hline & Toyota & Nissan & Suzuki \\
\hline Wald statistic & $9.1834 * * *$ & 0.0059 & $2.9262 *$ \\
\hline \multirow[t]{2}{*}{$p$-value } & 0.0024 & 0.9388 & 0.0872 \\
\hline & Mitsubishi & Mazda & Honda \\
\hline Wald statistic & 0.9234 & $16.9052 * * *$ & $6.9878 * * *$ \\
\hline$p$-value & 0.3366 & 0.0000 & 0.0082 \\
\hline \multicolumn{4}{|c|}{ Panel B. Results based on the ML estimators of a non-linear CAPM } \\
\hline & Toyota & Nissan & Suzuki \\
\hline Wald statistic & $13.4738 * * *$ & 0.0230 & 1.4253 \\
\hline \multirow[t]{2}{*}{$p$-value } & 0.0002 & 0.8795 & 0.2325 \\
\hline & Mitsubishi & Mazda & Honda \\
\hline Wald statistic & $10.3485 * * *$ & $12.4556 * * *$ & $5.3123 * *$ \\
\hline$p$-value & 0.0013 & 0.0004 & 0.0212 \\
\hline
\end{tabular}

Notes: This table shows the results of the Wald tests, where the null hypothesis is that the beta value equals one and the alternative hypothesis is that the beta value does not equal one. $* * * * *$, and $*$ indicate the statistical significance of the Wald test statistic at the $1 \%, 5 \%$, and $10 \%$ levels, respectively.

\section{Conclusions}

This paper quantitatively investigated a non-linear CAPM by using monthly stock returns of six major automobile industry firms in Japan. We consider that our empirical results as to the Japanese representative automobile industry firms supplied useful case study results not only for understanding the Japanese stock market but also for considering other international stock markets. In order to clearly inform the work and effectiveness of our non-linear CAPM, we attempted to explain our results by focusing on the two stock returns of fatter-tailed Mitsubishi and normally-distributed Honda in this study. However, it is emphasized that all results as to the other four Japanese automobile firms analyzed and exhibited in this study were also informative and consistently demonstrated the effectiveness of our non-linear CAPM applications.

More specifically, applying the maximum likelihood method, we obtained the following interesting findings. (1) First, in the case where the distribution of stock return has a fat-tail, the application of our non-linear CAPM was highly effective. As the parameters of our non-linear CAPM well captured fat-tailed stock return distributions, the non-linear model estimations derived reliable estimates of the CAPM beta values. This was shown particularly in the case of the stock return of Mitsubishi Motors in this study.

(2) Second, in the case where the stock return is normally distributed, we found that our non-linear CAPM was also effective. Since the parameters of our non-linear CAPMs also captured normally distributed stock returns by adjusting their degrees of freedom parameter values, the non-linear model estimations similarly derived reliable CAPM beta estimates as the standard linear CAPM estimations. In this paper, this was the case of the stock return of Honda.

(3) Furthermore, performing the Wald tests based on the estimators from the standard CAPM and those from a non-linear CAPM, we found that in the case where the distribution of stock return has a fat-tail, the Wald test result based on the estimators from our non-linear CAPM shall be more reliable than that based on the standard CAPM estimators. In this study, this was the case of Mitsubishi Motors. We further consider that in the case of normally distributed stock returns, the Wald test based on our non-linear CAPM is also reliable because the degrees of freedom parameter of the model can be adjusted for the normal distribution. In this paper, this was confirmed in the case of Honda. It is noted that the above interesting and useful findings are the contributions of this study.

Finally, as above, our quantitative investigations conducted in this paper suggest the interesting and important implication. Namely, both for normally distributed and fat-tailed stock returns, our non-linear CAPM applications are effective. Therefore, our results clearly suggest that in practice, it is better to apply non-linear CAPMs to derive the beta values to carefully capture the market risks of the stocks. As another implication, we further suggest that the sensitivities of the stocks for other risk factors in financial markets can be effectively derived by extending our methods. Based on these important results and implications suggested by this study, extended research by applying similar approaches by using different data sets and different models is important; and it is considered to be one of our future works. 


\section{Acknowledgements}

The author firstly thanks this journal for its kind repeated article invitation. In addition, the author greatly thanks Angelia Evelyn for her kind editorial assistance to this paper. Moreover, the author also thanks anonymous reviewers for their supportive and constructive comments on this paper. The author also appreciates the Japan Society for the Promotion of Science Grant-in-Aid for Scientific Research and the Chuo University Personal Research Grant for their generous financial assistance to my research. Finally, I deeply thank all the Editors of this journal for their kind attention to my paper.

\section{References}

Arısoy, Y. E., Altay-Salih, A., \& Akdeniz, L. (2015). Aggregate volatility expectations and threshold CAPM. North American Journal of Economics and Finance, 34, 231-253. http://dx.doi.org/10.1016/j.najef.2015.09.013

De Moor, L., \& Sercu, P. (2015). Measuring the impact of extreme observations on CAPM alphas: Some methodological issues. Finance Research Letters, 15, 1-10. http://dx.doi.org/10.1016/j.frl.2014.05.002

Messis, P., \& Zapranis, A. (2014). Herding towards higher moment CAPM, contagion of herding and macroeconomic shocks: Evidence from five major developed markets. Journal of Behavioral and Experimental Finance, 4, 1-13. http://dx.doi.org/10.1016/j.jbef.2014.09.002

Moussa, A. M., Kamdem, J. S., Shapiro, A. F., \& Terraza, M. (2014). CAPM with fuzzy returns and hypothesis testing. Insurance: Mathematics and Economics, 55, 40-57. http://dx.doi.org/10.1016/j.insmatheco.2013.11.001

Sharpe, W. F. (1964). Capital asset prices: A theory of market equilibrium under conditions of risk. Journal of Finance, 19, 425-442. http://dx.doi.org/10.2307/2977928

Tsuji, C. (2012). Positive return premia in Japan. Quantitative Finance, 12, 345-367. http://dx.doi.org/10.1080/14697688.2010.541485

Vendrame, V., Tucker, J., \& Guermat, C. (2016). Some extensions of the CAPM for individual assets. International Review of Financial Analysis, 44, 78-85. http://dx.doi.org/10.1016/j.irfa.2016.01.010

\section{Copyrights}

Copyright for this article is retained by the author(s), with first publication rights granted to the journal.

This is an open-access article distributed under the terms and conditions of the Creative Commons Attribution license which permits unrestricted use, distribution, and reproduction in any medium, provided the original work is properly cited. 